\title{
Numerical simulation of the three-dimensional structure and dynamics of the non-magnetic solar chromosphere
}

\author{
S. Wedemeyer ${ }^{1,2}$, B. Freytag ${ }^{3}$, M. Steffen ${ }^{4}$, H.-G. Ludwig ${ }^{5}$, and H. Holweger ${ }^{1}$ \\ ${ }^{1}$ Institut für Theoretische Physik und Astrophysik, Universität Kiel, 24098 Kiel, Germany \\ 2 Kiepenheuer-Institut für Sonnenphysik, Schöneckstrasse 6, 79104 Freiburg, Germany \\ 3 Department for Astronomy and Space Physics, Uppsala University, Box 515, 75120 Uppsala, Sweden \\ 4 Astrophysikalisches Institut Potsdam, An der Sternwarte 16, 14482 Potsdam, Germany \\ ${ }^{5}$ Lund Observatory, Box 43, 22100 Lund, Sweden
}

Received 3 February 2003 / Accepted 30 October 2003

\begin{abstract}
Three-dimensional numerical simulations with $\mathrm{CO}^{5} \mathrm{BOLD}$, a new radiation hydrodynamics code, result in a dynamic, thermally bifurcated model of the non-magnetic chromosphere of the quiet Sun. The 3D model includes the middle and low chromosphere, the photosphere, and the top of the convection zone, where acoustic waves are excited by convective motions. While the waves propagate upwards, they steepen into shocks, dissipate, and deposit their mechanical energy as heat in the chromosphere. Our numerical simulations show for the first time a complex 3D structure of the chromospheric layers, formed by the interaction of shock waves. Horizontal temperature cross-sections of the model chromosphere exhibit a network of hot filaments and enclosed cool regions. The horizontal pattern evolves on short time-scales of the order of typically $20-25 \mathrm{~s}$, and has spatial scales comparable to those of the underlying granulation. The resulting thermal bifurcation, i.e., the co-existence of cold and hot regions, provides temperatures high enough to produce the observed chromospheric UV emission and - at the same time - temperatures cold enough to allow the formation of molecules (e.g., carbon monoxide). Our 3D model corroborates the finding by Carlsson \& Stein (1994) that the chromospheric temperature rise of semi-empirical models does not necessarily imply an increase in the average gas temperature but can be explained by the presence of substantial spatial and temporal temperature inhomogeneities.
\end{abstract}

Key words. Sun: chromosphere - hydrodynamics - radiative transfer

\section{Introduction}

Three-dimensional, time-dependent radiation hydrodynamics simulations of solar and stellar surface convection have now reached a level of sophistication which goes far beyond that of idealised numerical experiments, and allows a direct confrontation of such models with real stars (e.g., Stein \& Nordlund 1998; Asplund et al. 2000; Freytag et al. 2002; Ludwig et al. 2002). Extending this kind of simulation to include the low chromosphere, it is possible to study - in a single model and based on first principles - the generation of waves by the convective flow as well as the wave propagation and dissipation in the higher layers. Extended simulations of this type may then be utilised to explore the hitherto poorly understood 3D thermal structure and dynamics of the non-magnetic chromospheric internetwork regions, and to obtain an independent theoretical estimate of the amount of chromospheric heating due to acoustic waves.

A strong motivation for three-dimensional time-dependent modelling arises from the need to reconcile apparently contradictory solar observations: carbon monoxide absorption lines

Send offprint requests to: $\mathrm{S}$. Wedemeyer, e-mail: wedemeyer@kis.uni-freiburg.de imply gas temperatures as low as $\approx 3700 \mathrm{~K}$ in the chromosphere of the quiet Sun (see Noyes \& Hall 1972; Ayres \& Testerman 1981; Solanki et al. 1994; Uitenbroek et al. 1994; Uitenbroek 2000a; Ayres 2002, and references therein), whereas chromospheric UV emission features require much higher temperatures at the same heights (e.g., Ayres \& Linsky 1976; Carlsson et al. 1997).

Semi-empirical models which have been constructed based on UV and microwave observations (e.g., Vernazza et al. 1981, hereafter VAL; Maltby et al. 1986; Fontenla et al. 1993, hereafter FAL) commonly feature a temperature minimum of $T_{\min } \approx 4200-4400 \mathrm{~K}$ at a height of $z \approx 500 \mathrm{~km}$ above optical depth unity and an outwardly increasing temperature above. On the other hand, models based on CO observations (e.g., Wiedemann et al. 1994) show a monotonic decrease of temperature with height.

These conflicting observations and the inferred representative models have led to a controversy about the nature of the chromosphere of the non-magnetic quiet Sun which is going on for many years now (see, e.g., Kalkofen 2001): is the chromosphere of the average quiet Sun a time-dependent phenomenon with a mostly cool background and large temperature fluctuations due to upward propagating shock waves? Or is it 
persistent and always hot with only small temperature fluctuations? In short: is the non-magnetic solar chromosphere hot or cool?

A large number of observations show that the chromosphere of the quiet Sun is indeed a very dynamic phenomenon (e.g., Carlsson et al. 1997; Muglach \& Schmidt 2001; Krijger et al. 2001; Wunnenberg et al. 2002). Obviously, static one-dimensional models can only describe selected timeaveraged properties. More realistic modelling should therefore be time-dependent.

Starting in the late 1960 s, the pioneering work on 1D time-dependent numerical models of chromospheric heating by acoustic and magneto-hydrodynamic waves is due to Ulmschneider and collaborators. In a long series of papers (e.g., Ulmschneider 1971; Ulmschneider \& Kalkofen 1977; Ulmschneider et al. 1978; Muchmore \& Ulmschneider 1985; Ulmschneider et al. 1987; Ulmschneider 1989; Cuntz et al. 1994), they studied in detail the chromospheric energy balance between dissipation of prescribed short-period (mostly monochromatic) acoustic waves and radiative emission. In their models, the acoustic energy flux is supplied by a piston acting as a lower boundary condition. Assuming that the generation of acoustic waves by the "turbulent" flows in the upper convection zone can be described by the Lighthill-Stein theory (Lighthill 1952; Stein 1967, 1968; Musielak et al. 1994; Ulmschneider et al. 1996, 1999), they compute dynamic chromospheric models not only for the Sun but also for a sample of main-sequence stars and giants. Based on these models, they conclude that the observed "basal flux" from the chromospheres of late-type stars (Schrijver 1987; Rutten et al. 1991) is fully attributable to the dissipation of acoustic wave energy (Buchholz et al. 1998), and that the observed variation of chromospheric emission can be explained by the additional heating of magnetohydrodynamic shock waves (Ulmschneider et al. 2001).

The detailed radiation hydrodynamics simulations by Carlsson \& Stein (1994, 1995, 1997, hereafter CS) are another prominent example of sophisticated 1D timedependent modelling. These authors successfully explained the $\mathrm{Ca} \mathrm{II} \mathrm{H}_{2 \mathrm{v}}$ bright points as a result of propagating shock waves. In their model, the waves are excited by a piston which is driven by a velocity variation derived from observed oscillations at the photospheric level. Instead of a temperature minimum and a monotonic temperature increase above, as characteristic of the VAL and FAL models, CS find a chromosphere with a mostly cool background and large temperature fluctuations due to upward propagating shocks. Even more remarkable is the fact that they are able to reproduce the rise of the radiation temperature without an increase of the mean gas temperature. Basic reasons are the nonlinear temperature dependence of the Planck function in the UV and the extreme temperature peaks associated with the shock waves. This led CS to the conclusion that the chromosphere of the quiet Sun is not persistent but a spatially and temporally intermittent phenomenon which - if averaged over space and time - is mostly cool and not hot.

Although the one-dimensional models of the non-magnetic solar chromosphere mentioned above are highly elaborate, including a fully time-dependent $\mathrm{H}$ ionisation and detailed
NLTE radiative transfer, they suffer from the need for an external prescription of the wave excitation, and of course they cannot account for horizontal inhomogeneities and the associated effect of dynamic cooling on the atmospheric energy balance.

In this regard, the three-dimensional self-consistent modelling by Skartlien et al. (2000) can be considered as a major improvement. The idea of Skartlien and co-workers was to extend the standard radiation hydrodynamics simulations of the solar granulation (Stein \& Nordlund 1998) into the chromosphere, where local thermodynamic equilibrium (LTE) is known to be a poor approximation. In order to adapt it to chromospheric conditions, Skartlien (2000) upgraded the radiative transfer part of the Nordlund-Stein code by implementing an iterative method to treat coherent isotropic scattering in $3 \mathrm{D}$. The simulations enabled Skartlien et al. to analyse the generation, propagation, and dissipation of acoustic waves in three dimensions. The main emphasis of their study was on the excitation of transient wave emission resulting from the collapse of small granules, and the dynamic response of the chromospheric layers to such acoustic events.

In the present paper, we present similar time-dependent 3D models which extend from the upper convection zone to the middle chromosphere. The radiation hydrodynamics simulations are performed with $C \mathrm{O}^{5} \mathrm{BOLD}$, a new radiation hydrodynamics code developed by B. Freytag and M. Steffen (Freytag et al. 2002). In this exploratory simulation, we treat the radiative transport in LTE with grey opacities (see Sect. 2.2 and discussion in Sect. 5). This simplification allows us to work at a significantly higher spatial resolution $(140 \times 140 \times 200$ cells $)$ than Skartlien et al. $(32 \times 32 \times 100$ grid $)$. We find that the $3 \mathrm{D}$ structure of the non-magnetic chromospheric layers is characterised by a complex pattern of interacting shocks, forming a network of hot filaments and enclosed cool "bubbles". This chromospheric pattern and its implications are chosen as major subject of this paper since the topology and the dynamics of the pattern are likely not to be too sensitive to the LTE simplification. We conclude that the low chromosphere exhibits a prominent thermal bifurcation: hot and cool regions exist side by side. Surprisingly, this small-scale (non-magnetic) network was not mentioned by Skartlien et al.; presumably, it was not noticed due to the poor (horizontal) spatial resolution of their numerical model.

In Sect. 2 we will give a short overview of the numerical details of $\mathrm{CO}^{5} \mathrm{BOLD}$. The 3D model is described in Sect. 3, followed by the results in Sect. 4. Finally, a discussion and conclusions are presented in Sects. 5 and 6, respectively.

\section{The radiation hydrodynamics code $\mathrm{CO}^{5} \mathrm{BOLD}$}

CO ${ }^{5}$ BOLD solves the time-dependent hydrodynamic equations coupled with the radiative transfer equation for a fully compressible, chemically homogeneous plasma in a constant gravitational field in two or three spatial dimensions. Operator splitting separates Eulerian hydrodynamics, 3D tensor viscosity, and radiation transport. Magnetic fields are not included so far, restricting this version of $\mathrm{CO}^{5} \mathrm{BOLD}$ to internetwork regions.

The most important properties of the code are described below (see also Freytag et al. 2002; Wedemeyer 2003). A more 
detailed paper on the code itself is in preparation (Freytag, in prep.).

\subsection{Hydrodynamics}

The relations for the conservation of mass, momentum, and energy are solved on a fixed Cartesian grid allowing spatially non-equidistant meshes. Directional operator splitting transforms the $2 \mathrm{D} / 3 \mathrm{D}$ problem into $1 \mathrm{D}$ sub steps which then can be treated with a fast approximate Riemann solver (Roe 1986). The scheme is modified to account for a realistic equation of state and an external gravity field.

Additionally, a small amount of tensor viscosity is added in a separate sub step. Although the hydrodynamics scheme is stable enough to handle 1D and most multi-dimensional problems, there are special multi-dimensional cases which require an additional tensor viscosity to ensure stability. Such cases occur, e.g., near strong shocks which are aligned with the grid (Quirk 1994). Our numerical scheme has proven to be very robust in handling shocks, which is important when modelling chromospheric conditions.

\subsection{Radiation transport}

The equation of radiative transfer is solved applying long characteristics ("rays"). A large number of rays traverse the computational box under different azimuthal and inclination angles. Independently along each ray, the radiative transfer equation is solved with a modified Feautrier scheme. The radiation transport is treated in strict LTE so far. In this work, a grey (frequency-independent) radiation transport with realistic opacities is used (see Sect. 2.3). The applied scheme is wellsuited for the lower layers (convection zone and photosphere), but clearly requires further improvements for chromospheric conditions where substantial deviations from LTE prevail and the UV radiative transfer is dominated by scattering. See also the discussion in Sect. 5.

\subsection{Equation of state and opacities}

The equation of state takes into account partial ionisation of $\mathrm{H}$ and $\mathrm{He}$, as well as formation and dissociation of $\mathrm{H}_{2}$, assuming thermodynamic equilibrium. It is solved by interpolation in a table which is computed in advance for a prescribed chemical composition of hydrogen, helium, and a representative metal. The table consists of two-dimensional arrays as functions of density and internal energy.

For the model presented in this work we used a Rosseland mean opacity look-up table which has been compiled and processed based on data of OPAL for temperatures above $12000 \mathrm{~K}$ (Iglesias et al. 1992) and PHOENIX for temperatures below $12000 \mathrm{~K}$ (Hauschildt et al. 1997, and references therein). The table provides the opacity as a function of temperature and gas pressure.

Although a large number of atomic lines and molecular features are formally taken into account in the construction of the opacity table, it is clear that the stronger lines are not properly represented when computing the grey opacity according to the Rosseland averaging procedure. Consequently, the stronger spectral features are essentially ignored in the present approach (see also Sect. 5).

\subsection{Boundary conditions}

Located deep in the convectively unstable layers, the lower boundary is open, i.e., material is allowed to flow in and out of the computational box. The inflow of material is constrained to ensure a vanishing total mass flux across the lower boundary so that the total mass in the computational volume is preserved - aside from smaller gains or losses across the upper boundary. The entropy of inflowing material is a prescribed parameter, and indirectly controls the effective temperature of a model. The vertical derivative of the velocity components is zero. The pressure in the bottom layer is kept close to planeparallel by artificially reducing horizontal pressure fluctuations towards zero with a prescribed time constant.

At the upper transmitting boundary the vertical derivative of the velocity components and of the internal energy are zero; the density is assumed to decrease exponentially above the top boundary. Material can flow into the computational box if the velocity at the boundary is directed downwards. The temperature of the inflowing material is then altered towards a temperature $T_{\text {top }}$ on a characteristic time scale of typically a few seconds. This simple boundary condition turns out to be stable and allows (shock) waves to leave the computational box without noticeable reflections. Moreover, we have chosen the location of the upper boundary such that it is far away from the regions which are of particular interest in this work.

The lateral boundary conditions are periodic.

\section{The 3D model}

The 3D model consists of horizontally 140 grid points $(x, y)$ with a constant resolution of $40 \mathrm{~km}$, leading to a horizontal size of $5600 \mathrm{~km}$ which corresponds to an angle of $\approx 7^{\prime \prime} .7$ in groundbased observations. The total vertical height is $3110 \mathrm{~km}$, reaching from the upper convection zone at $z=-1400 \mathrm{~km}$ to the middle chromosphere at a height of $z=1710 \mathrm{~km}$. The origin of the geometric height scale $(z=0 \mathrm{~km})$ corresponds to the temporally and horizontally averaged Rosseland optical depth unity. In the following we refer to the photosphere always as the layer between $0 \mathrm{~km}$ and $500 \mathrm{~km}$ in model coordinates, and to the chromosphere as the layer above. The 200 vertical grid points are non-equidistant, with a resolution of $46 \mathrm{~km}$ at the bottom which decreases with height down to a constant distance of $12 \mathrm{~km}$ for all layers above $z=-270 \mathrm{~km}$. The computational time step is typically 0.1 to $0.2 \mathrm{~s}$.

As an initial model, we extended an already evolved model which reached up to the top of the photosphere. The temperature and density stratification for the new grid cells were calculated under the assumption of hydrostatic equilibrium. Interestingly, the further evolution of the model does not depend strongly on the initial condition because the chromosphere turns out to be highly dynamical on short time-scales. 

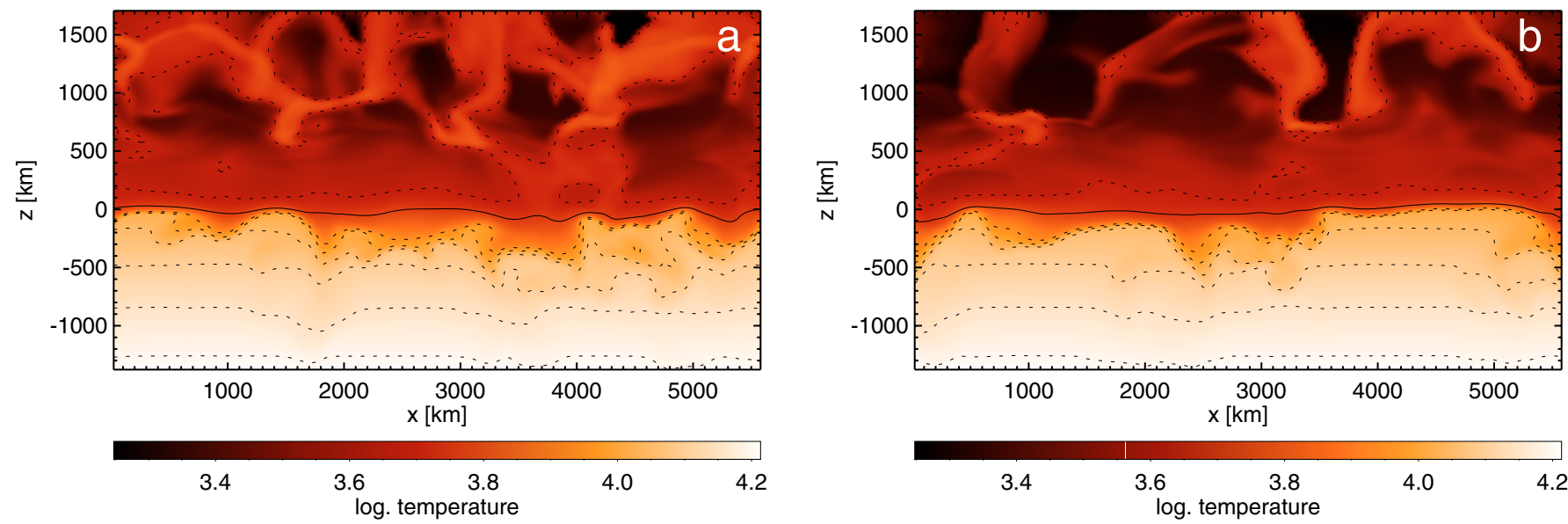

Fig. 1. Logarithmic temperature in vertical 2D slices taken from the 3D model at different horizontal positions (a) $y=1540 \mathrm{~km}$, b) $y=$ $2820 \mathrm{~km}$ ): top of the convection zone, photosphere, and low/middle chromosphere with propagating shock waves. The solid line marks the height for optical depth unity. The dotted lines are contours for $\log T=3.7,3.95,4.00,4.05, \ldots, 4.20$ (top to bottom). The temperature ranges from $\approx 16400 \mathrm{~K}$ to $\approx 5200 \mathrm{~K}$ in the convection zone (i.e., below $z=0 \mathrm{~km}$ ) and decreases to $\approx 3000 \mathrm{~K}$ in the photosphere and even down to $\approx 1800 \mathrm{~K}$ in the chromosphere.

After only a few minutes of simulation time the initial chromosphere already formed the typical structures which we will discuss below.

However, the first $170 \mathrm{~min}$ of the simulation sequence are not used for data analysis to ensure that the model has sufficiently relaxed. The results presented in this work are based on another $151 \mathrm{~min}$ of simulation time.

\section{Results}

\subsection{Structure of the model atmosphere}

Figures 1-3 show the temperature in vertical and horizontal slices of the 3D model which is described in Sect. 3. The data for Figs. 1-2 are taken from the same time step. Figure 2 illustrates the depth-dependence of the structure of the model atmosphere by means of 2D temperature slices at various geometrical heights. The same figure also shows synthetic images of the emergent continuum intensity at $\lambda=5500 \AA$ and $\lambda=1600 \AA$ which were computed subsequent to the simulation for the selected time step. For these calculations LTE radiative transfer was assumed. We used pure continuum opacities (dominated by Si I b-f absorption at $1600 \AA$ ) taken from the Kiel spectrum synthesis package LINFOR.

Obviously, there are striking differences between the horizontal patterns in the photosphere and the layers above. The temperature at the bottom of the photosphere (Fig. 2a) reveals the granulation which comes out more clearly in the intensity image for $\lambda=5500 \AA$ (Fig. $2 \mathrm{~g}$ ). The granulation is very similar to observations in various aspects like shape, size distribution, and lifetime of the granules, indicating that in the lower part of the model the physics are realistically represented (Wedemeyer 2003). Only $250 \mathrm{~km}$ above, a reversed granulation pattern appears (Fig. 2b): the inner parts of the granules are dark due to the rapid cooling of the ascending gas, and bright rims (note the double structure) appear at the edges of the granules, representing hot shocked gas being directed into the intergranular lanes.
Higher up, the model chromosphere is characterised by a network of hot matter and small-scale hot spots on a cool background as can be seen in the horizontal cross-sections in Figs. $2 \mathrm{~d}-\mathrm{f}$. The pattern is a result of interaction of propagating hydrodynamic shock waves which are an ubiquitous phenomenon in the model chromosphere. The shock fronts are usually inclined, so a horizontal cut through the temperature field shows a filamentary structure. There is also a clear signature of oscillations with periods in the 3-min range (see Fig. 4). Shock waves are present at all time steps, mostly several at the same time (Figs. 1-3). The waves propagate in the vertical as well as in the horizontal direction and interfere with each other, compressing and heating the gas in the filaments (see Sect. 4.2).

As a consequence of the correlation between convective motions and the excitation of acoustic waves, the spatial scale of the pattern is comparable to that of the underlying granulation.

The network-like pattern appears more subtle in the UV continuum intensity at a wavelength of $\lambda=1600 \AA$ (Fig. 2h). Rather, a small area of enhanced emission stands out of an otherwise dark background. This is caused by the highly non-linear temperature response of the Planck function in the UV. Hence, the hot gas, which is connected to the propagating shock waves, contributes by far more to the emergent UV continuum intensity than the cool regions. Note that for more realistic results, scattering and line blocking must be taken into account.

Due to the ongoing propagation of the waves the pattern changes continuously (see Fig. 3) on time-scales which are much shorter than derived for the granulation. We calculated autocorrelation times for sequences of horizontal temperature slices and determined height-dependent pattern evolution time scales as the time lags for which the autocorrelation decreased to a value of $1 / e$. At chromospheric heights the characteristic time scales are as short as 20-25 s whereas the same analysis produces time scales of $\gtrsim 120 \mathrm{~s}$ at the bottom of the photosphere $(z=0)$. Using the emergent grey intensity, which 



Fig. 2. Temperature in horizontal $2 \mathrm{D}$ slices at different heights in the photosphere at $z=0 \mathrm{~km}, 250 \mathrm{~km}$, and $500 \mathrm{~km}(\mathbf{a})-\mathbf{c})$ ), and in the chromosphere at $z=750 \mathrm{~km}, 1000 \mathrm{~km}$, and $1250 \mathrm{~km}(\mathbf{d})-\mathbf{f})$ ). Panels $\mathbf{g}$ ) and h) show the emergent continuum intensity at $\lambda=5500 \AA$ and $\lambda=1600 \AA$, respectively.

renders the low photosphere, instead of the gas temperature leads to $\sim 200 \mathrm{~s}$. The difference between temperature and intensity result can be understood if one considers that structures also move up and down, for instance, due to oscillations.
Consequently, the pattern at a fixed geometrical height changes more quickly than visible in the corresponding intensity. Furthermore, spatial smearing of the pattern, i.e., reducing the image resolution to values caused by observational seeing 

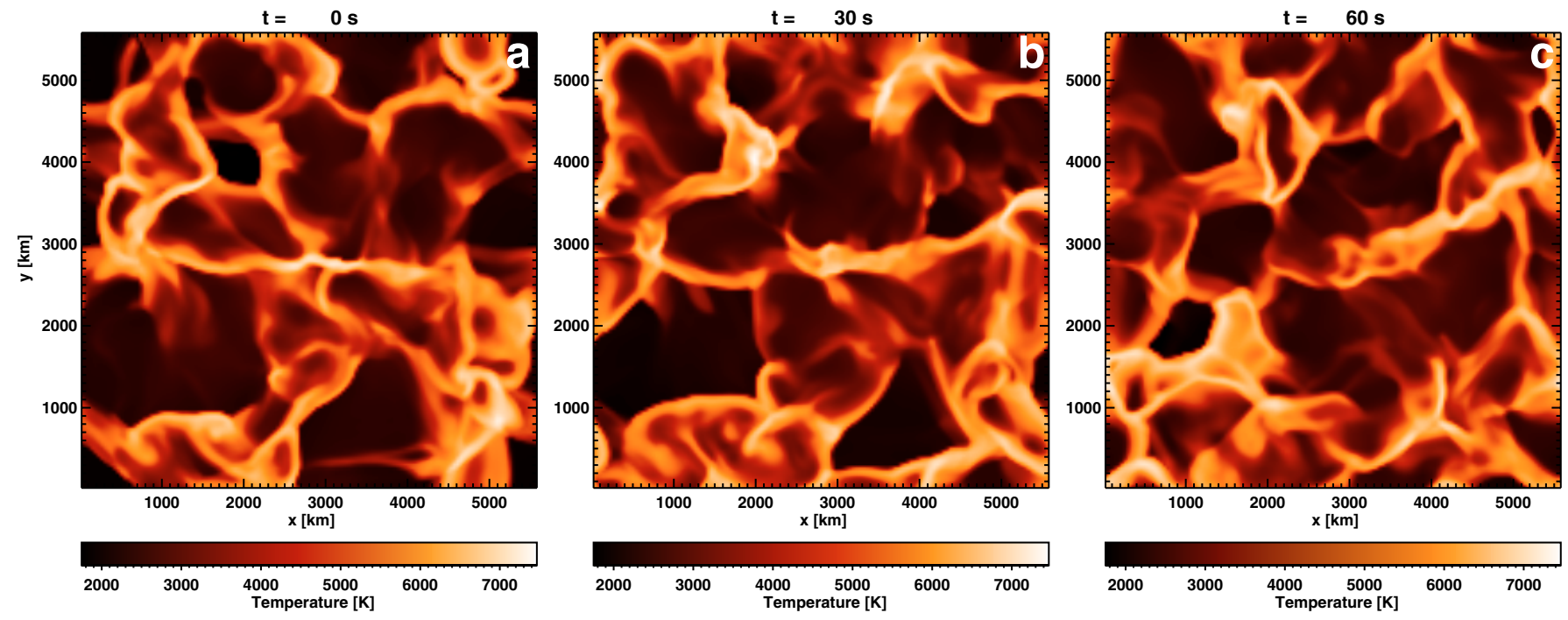

Fig. 3. Temperature in horizontal $2 \mathrm{D}$ slices at $z=1000 \mathrm{~km}$ for a short time sequence $(\Delta t=30 \mathrm{~s})$.



Fig. 4. Variation of relative velocity power with height. At each height the power spectrum of the horizontal average of the vertical velocity was integrated over the frequency intervals which are specified on the left.

conditions, produces longer time scales. This should be kept in mind when comparing the theoretical results with empirical data.

\subsection{Waves, oscillations, and shocks}

Acoustic waves are excited by various processes concentrated in the uppermost layers of the solar convection zone. Excitation processes have been investigated by means of hydrodynamical modelling by Skartlien et al. (2000), Nordlund \& Stein (2001), and Stein \& Nordlund (2001). Skartlien et al. study the collapse of small granules which leads to transient wave emission. Nordlund \& Stein focus on the interaction of convection with resonant oscillatory modes to derive an estimate of the power input into the solar 5 min oscillations. Like the afore mentioned authors, we observe in our model the excitation of both propagating and standing acoustic waves. The standing waves are the model analogs to the solar 5 min oscillations. Together with the propagating waves they generate a complex interference pattern in the photospheric and chromospheric layers, where shocks are frequently formed.

Figure 4 illustrates the distribution of power among radial oscillations as a function of height. Fourier spectra were calculated for a $151 \mathrm{~min}$ long time sequences of the horizontally averaged vertical velocity component at each height independently, and integrated over frequency bands roughly centred around periods of $5 \mathrm{~min}$ and $3 \mathrm{~min}$. Figure 4 shows that the dominant contribution to the velocity power shifts from the 5 -min band to the 3-min band at around $z \sim 1200 \mathrm{~km}$. We find no significant power in the low frequency band (periods larger than $\sim 420 \mathrm{~s}$ ), while the high frequency band (periods below $\sim 140 \mathrm{~s}$ ) contributes some power in the higher layers.

The absolute energy of the oscillatory motions (not shown) decreases in all bands with increasing height. The largest energies are found in the deepest layers, indicating that the excitation of the oscillations takes place in the convection zone for all frequencies. The 5-min band lies below the acoustic cutoff frequency $(\sim 5.5 \mathrm{mHz})$ rendering these waves evanescent while in the 3-min band some frequencies allow propagating waves. This implies a stronger damping in the 5-min band, and explains why the "3-min" oscillations dominate in the chromosphere: the decline of energy with height is more pronounced in the 5-min band than in the 3-min band. A localised non-linear process converting oscillatory energy in the 5-min band into energy in the 3-min band is not readily apparent.

Examples of propagating waves and shock formation are shown in Fig. 5. The example of the left-most column (Fig. 5a) is displayed more quantitatively in Fig. 6 for further discussion below. It shows the case of a rather localised shock which was triggered by pressure disturbances emerging from the downflow region visible in the deeper layers. The formation of a spherically shaped shock is a frequent pattern. The spherical 



Fig. 5. Formation and propagation of shock fronts: each column shows a time sequence of vertical slices (temperature) taken from different positions and times of the 3D model. a) (left column) arch-like/spherical wave, b) (middle column) plane wave, c) (right column) waves excited by merging downdrafts. Note the different time steps which are quoted in the lower left corners. The temperature is colour-coded for the range $T=2000 \mathrm{~K}$ to $T=7500 \mathrm{~K}$. Additional contour lines are present for $T=5000 \mathrm{~K}$ (dotted), and $T=7500 \mathrm{~K}, 9000 \mathrm{~K}$, and $10000 \mathrm{~K}$ (all solid).

shock front appears as an upward travelling arch-like feature in our 2D cuts. The middle column in Fig. 5 shows an example of a front which is horizontally more extended. In movies such events appear often as if the front detaches over a broader area from the photospheric granulation pattern. It can extend over more than one granule and tends to preserve the shape of the granular pattern for some time. In the simulation, preferentially resonant modes of long horizontal wavelength are excited. They provide the horizontally coherent oscillations which are necessary to produce these extended horizontal wave fronts. The right-most column (Fig. 5c) shows the formation of shocks above merging downdrafts, i.e., downflows in the intergranular lanes. This kind of event corresponds to the collapse of small granules and has already been investigated in detail by Skartlien et al. (2000). From the vertical slices in Fig. 5c it can be seen how two downdrafts are advected horizontally and eventually merge, producing a stronger and more extended downdraft. During the process upward propagating waves are excited which may transform into shocks in higher layers. Moreover, a strong downdraft is often accompanied by shocks of a different nature. They come about by fast horizontal flows towards the downdraft. Shocks form where the flow is 


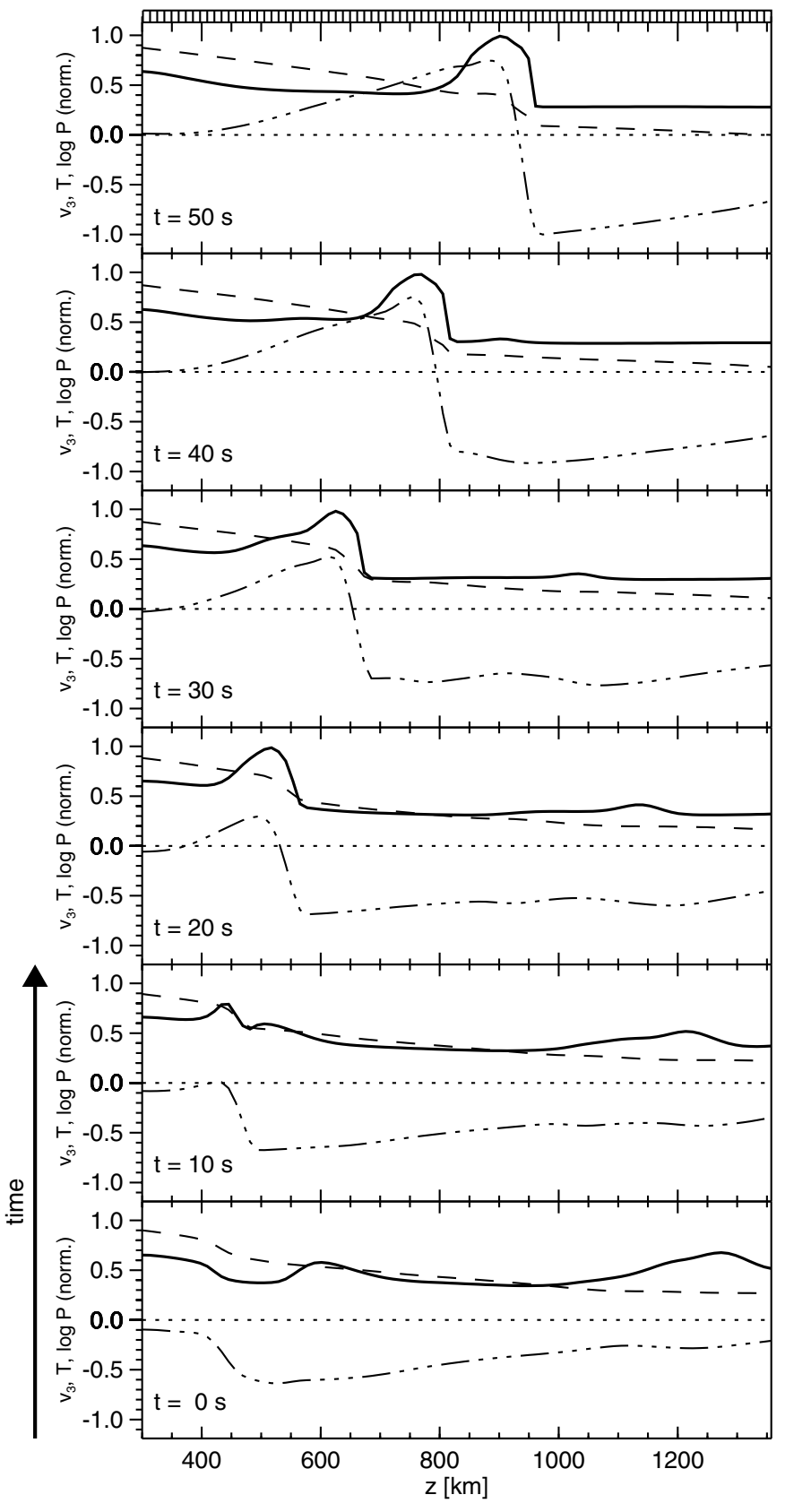

Fig. 6. Vertical profiles of the flow field shown in Fig. 5a at different times along the horizontal position $x=0 \mathrm{~km}$. We plot the temperature (solid), the vertical velocity component (triple-dot-dashed), and the logarithmic pressure (dashed) on a linear scale. The data range 0 to 1 corresponds to $0 \mathrm{~K}$ to $7000 \mathrm{~K}$ in temperature, -1.59 to 4.50 (cgs units) in the logarithmic pressure, and $0 \mathrm{~km} \mathrm{~s}^{-1}$ to $15 \mathrm{~km} \mathrm{~s}^{-1}$ in the vertical velocity component. The vertical grid is shown at the top of the figure.

turned into the downdraft. In Fig. 5c they are visible as roughly vertical features attached to the edges of a downdraft. These shocks interact with the shocks associated with the wave field (see frames at $60 \mathrm{~s}$ to $120 \mathrm{~s}$ ). Note that Fig. 5 shows particularly clean examples of the types of shock events encountered in the simulation. Usually, the pattern of shocks is very entangled, and often all features discussed before are present at the same time.
The wave depicted in Fig. 6 (see also Fig. 5a) is an extreme example as a positive vertical velocity of $v_{z} \approx 11 \mathrm{~km} \mathrm{~s}^{-1}$ is reached in the chromosphere. Most velocities are smaller. We find approximate upper limits for $95 \%$ of all upward directed vertical velocities, depending on height: $\approx 4.9 \mathrm{~km} \mathrm{~s}^{-1}$ at $z=$ $800 \mathrm{~km}$ and $\approx 7.0 \mathrm{~km} \mathrm{~s}^{-1}$ at $z=1000 \mathrm{~km}$. In contrast to onedimensional simulations, the waves in our 3D model do not only propagate in the vertical direction but also horizontally. At a height of $z=1000 \mathrm{~km}$ we find that $95 \%$ of all grid cells exhibit horizontal velocities of less than $\approx 12 \mathrm{~km} \mathrm{~s}^{-1}$ and $50 \%$ have values of $\approx 5 \mathrm{~km} \mathrm{~s}^{-1}$ and below.

An important point is illustrated in Fig. 6: shocks are preferentially formed in low-density material which is flowing down from above at high velocities. The material has been pushed upwards by a precursory wave and now falls back again. The shock front is travelling upstream into the down-flowing material. In extreme cases the downflowing material is close to free-fall conditions, and flow velocities exceed the local sound speed. The 1D simulations by Carlsson \& Stein (1997) exhibit a similar shock structure (see their Fig. 14). Judging from the same figure, Carlsson \& Stein find typically at most one well developed shock in the photospheric and chromospheric layers at any given instant in time. Looking at one particular vertical column in our 3D model we make a similar observation, finding typically one, sometimes two fronts. While in their piston-driven model Carlsson \& Stein derive the wave excitation semi-empirically from observed time sequences of photospheric oscillations, the shock frequency in our case is a natural outcome of the simulation. The spatial shock frequency translates into a temporal recurrence of shocks on a time scale of $2-3 \mathrm{~min}$ (see also Fig. 10).

\subsection{Thermal bifurcation}

Although the chromospheric pattern evolves on very short time scales (see Sect. 4.1), the general picture remains the same in time, i.e., the chromosphere appears as a network of hot matter with intermittent cool regions. This thermal bifurcation can be quantified via a height-dependent temperature histogram. For each horizontal slice in the model (constant height $z$ for each slice) a histogram of the temperature values is calculated for temperature bins of $\Delta T=100 \mathrm{~K}$. The result is shown in Fig. 7. In the photosphere, the temperature is distributed close to a mean value with only moderate deviations, whereas in the chromosphere, the distribution splits up into low and high temperatures. Again, this indicates the co-existence of a cool background and hot shocked material.

To facilitate a rough comparison with multi-component models (e.g., Ayres et al. 1986; Avrett 1995; Ayres \& Rabin 1996; Ayres 2002), we give approximate values for a hot and a cool component of our model chromosphere (intermediate values are neglected): above $z=800 \mathrm{~km}$ the hot temperature ridge in Fig. 7 peaks at $T_{\text {hot }}=5500-5900 \mathrm{~K}$, whereas the cool temperature peak decreases with height from $T_{\text {cool }}=2600 \mathrm{~K}$ at $z=800 \mathrm{~km}$ to $\approx 2000 \mathrm{~K}$ for the upper layers of the model chromosphere. Thus, the hot component is comparable to the temperatures in the semi-empirical models $\mathrm{C}$ by VAL and C' 

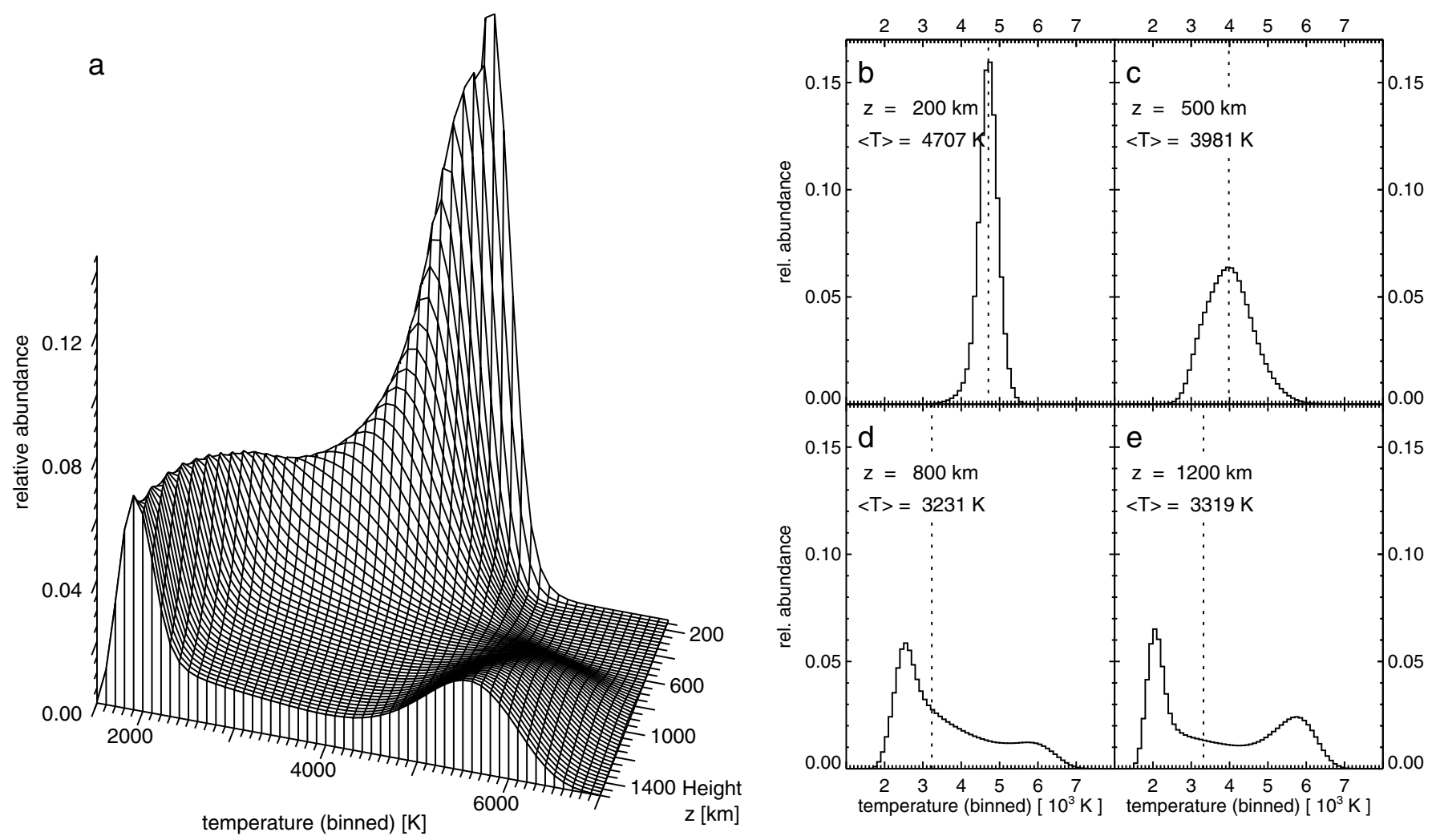

Fig. 7. Temperature histograms for the 3D model (151 min of simulation time). For each height step (and all time steps) the temperature values of all grid cells within the corresponding horizontal plane are sorted into temperature bins of $\Delta T=100 \mathrm{~K}$. The vertical axis denotes the relative abundance of grid cells within a temperature bin with respect to all cells at that height. a) Height-dependent histogram surface. b-c) Histograms at fixed heights in the photosphere and $\mathbf{d}-\mathbf{e}$ ) in the chromosphere. The dotted lines represent the corresponding mean temperature.

by Maltby et al. (1986) in the height range $800-1000 \mathrm{~km}$ and $900-1100 \mathrm{~km}$ for the model A by FAL, respectively. The cool component is much colder than COOLC by Ayres et al. (1986) and COOL0 by Ayres \& Rabin (1996). It is much more like COOL1 (Ayres 2002) around $z=800 \mathrm{~km}$ which, however, is only valid if there is a dominating warm component.

\subsection{Temperature stratification}

In this section we discuss the consequences of the thermal bifurcation for the average temperature stratification. The horizontally and temporally averaged gas temperature for the sequence of $151 \mathrm{~min}$ simulation time from our model (thin solid line in Fig. 8a) decreases with height until it reaches values between $3800 \mathrm{~K}$ and $3700 \mathrm{~K}$ above $z=730 \mathrm{~km}$, i.e., in the chromosphere. It does not show a notable temperature minimum nor a significant temperature increase in the chromosphere like it is the case in the semi-empirical models by VAL and FAL (see Fig. 8a). This is qualitatively similar to the mean gas temperature profile in the 1D simulation by Carlsson $\&$ Stein (1995) which also does not show a temperature increase (see Fig. 8a). However, we obtain chromospheric gas temperatures which are much lower than in the simulations by CS. In fact, our mean chromospheric gas temperature lies about $1000 \mathrm{~K}$ below the (grey) radiative equilibrium temperature of $4680 \mathrm{~K}$. The mean temperature stratification is roughly comparable to model COOLC by Ayres et al. (1986), which was constructed as the cool constituent in a multi-component model (see Fig. 8a, where we converted the original column mass density scale into a geometrical height scale on the basis of model $\mathrm{C}$ by VAL).

The semi-empirical models are based on spatially and temporally averaged intensities and thus refer to a static and homogeneous chromosphere. We note that the mean gas temperature from our model matches almost perfectly the semi-empirical models up to a height of $z \approx 500 \mathrm{~km}$. Above that height, the thermal bifurcation becomes increasingly significant, i.e., the temperature fluctuations become large (see Figs. 7 and 9). Clearly, the assumption of spatial and temporal homogeneity is not valid in the chromosphere, and any one-dimensional static description must fail.

CS pointed out that the chromospheric temperature rise in the semi-empirical models is only an artifact caused by the "temporal averaging of the highly nonlinear UV Planck function". Furthermore, CS confirmed this by calculating a temperature distribution for their dynamical model in a similar way as VAL. They adjusted a steady-state temperature stratification to reproduce the time-averaged continuum and line intensities as a function of wavelength which are a result of their dynamic simulation. The semi-empirical model derived in this way by $\mathrm{CS}$ is a much better fit to the models VAL and FAL (see Fig. 8a).

Since no wavelength-dependent intensities are available for our simulation (except for a few images similar to those shown 

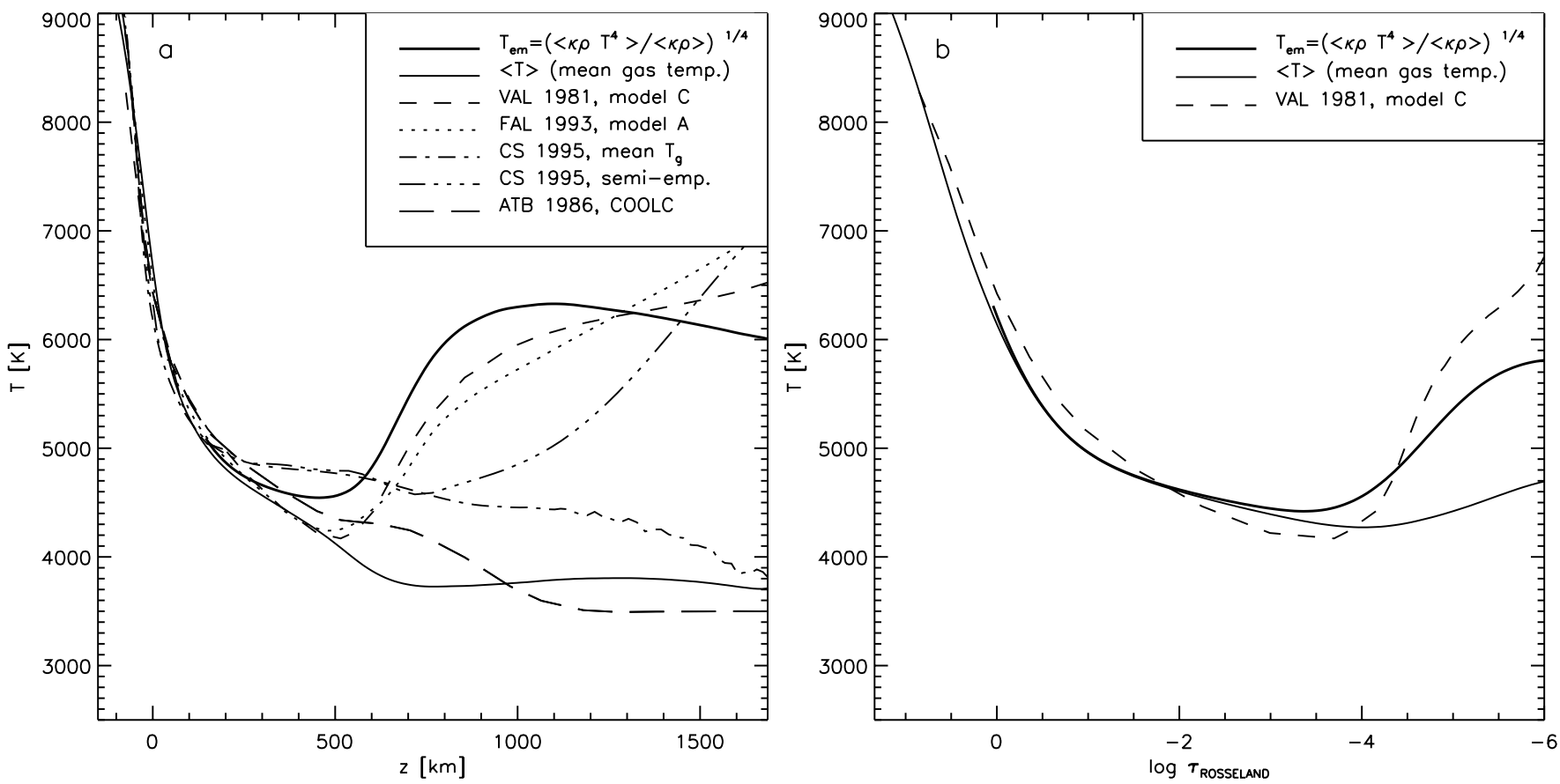

Fig. 8. Temperature stratifications of different models on a geometric height scale a) and on an optical depth scale b): horizontally and temporally averaged grey emissivity temperature and mean gas temperature for the 3D model, model C by Vernazza et al. (1981), model A by Fontenla et al. (1993), mean gas temperature and semi-empirical stratification of the dynamical model by Carlsson \& Stein (1995), and COOLC by Ayres et al. (1986).

in Figs. $2 \mathrm{~g}, \mathrm{~h}$ ), we calculated a qualitatively similar quantity, namely an "average grey emissivity temperature", by averaging the grey emissivity $\kappa \rho T^{4}$, where $\kappa$ is the opacity and $\rho$ the density. The corresponding emissivity temperature $T_{\mathrm{em}}$ is then evaluated as:

$T_{\mathrm{em}}(z)=\left\langle\left(\frac{\left\langle\kappa \rho T^{4}\right\rangle_{x, y}}{\langle\kappa \rho\rangle_{x, y}}\right)^{1 / 4}\right\rangle_{t}$.

the brackets $\langle.\rangle_{x, y},\langle.\rangle_{t}$ indicate horizontal and temporal averaging, respectively. The resulting average temperature profile, calculated on a geometrical scale (thick solid line in Fig. 8a) is indeed similar to model $\mathrm{C}$ by VAL and model A by FAL. It exhibits a temperature minimum at approximately the same height; the temperature values reached in the middle chromosphere are comparable. This qualitative match is better than expected from such a crude approximation. Thus, like CS we are able to produce an emissivity temperature stratification qualitatively similar to the semi-empirical models, without a significant increase in the mean gas temperature.

The averages presented so far are calculated on a geometrical height scale. In contrast, the average grey emissivity temperature and the simple arithmetic average shown in Fig. $8 \mathrm{~b}$ are calculated on the Rosseland optical depth scale which already incorporates the distribution of opacity and density. Hence, the emissivity temperature is given by $T_{\tau}^{4}$ averaged over surfaces of constant optical depth.

We note that the mean chromospheric gas temperature obtained from averaging on the optical depth scale are systematically higher (but still below the radiative equilibrium value) than those on the geometrical height scale; the minimum values differ by more than $500 \mathrm{~K}$. That is caused by the fact that fluctuations appear much smaller on surfaces of equal optical depth (see e.g., Uitenbroek 2000b). In a wave front the optical depth increases significantly. Thus, averaging on an optical depth scale is done on surfaces which are not plane but shaped by the spatial inhomogeneities while averaging on a geometrical height scale is done on strictly plane surfaces which cut through the inhomogeneities. Consequently, the temperature distribution on a surface for a particular optical depth differs from the one for a corresponding geometrical height, leading to different horizontal averages and thus different temperature stratifications.

\subsection{RMS-temperature fluctuations}

Here, we quantify the rms-temperature fluctuations which are another measure characterising the thermal structure. They are defined by

$\frac{\mathrm{d} T_{\mathrm{rms}}}{T_{0}}=\frac{\sqrt{\left\langle\left(T-T_{0}\right)^{2}\right\rangle_{x, y, t}}}{T_{0}}$

where $T_{0}=\langle T\rangle_{x, y, t}$ is the temporally and horizontally averaged temperature stratification. The quantity $\mathrm{d} T_{\text {rms }} / T_{0}$ has been calculated on a geometrical and on an optical depth scale for the same model sequence as in Sect. 4.4 (Fig. 9). It is strongly height-dependent as can also be seen directly from the horizontal slices in Fig. 2 for different heights and from the temporal temperature variation in Fig. 10. Obviously, the lower layers of the solar atmosphere in our model are relatively homogenous with only small temperature fluctuations, in contrast to the inhomogeneous chromosphere. 

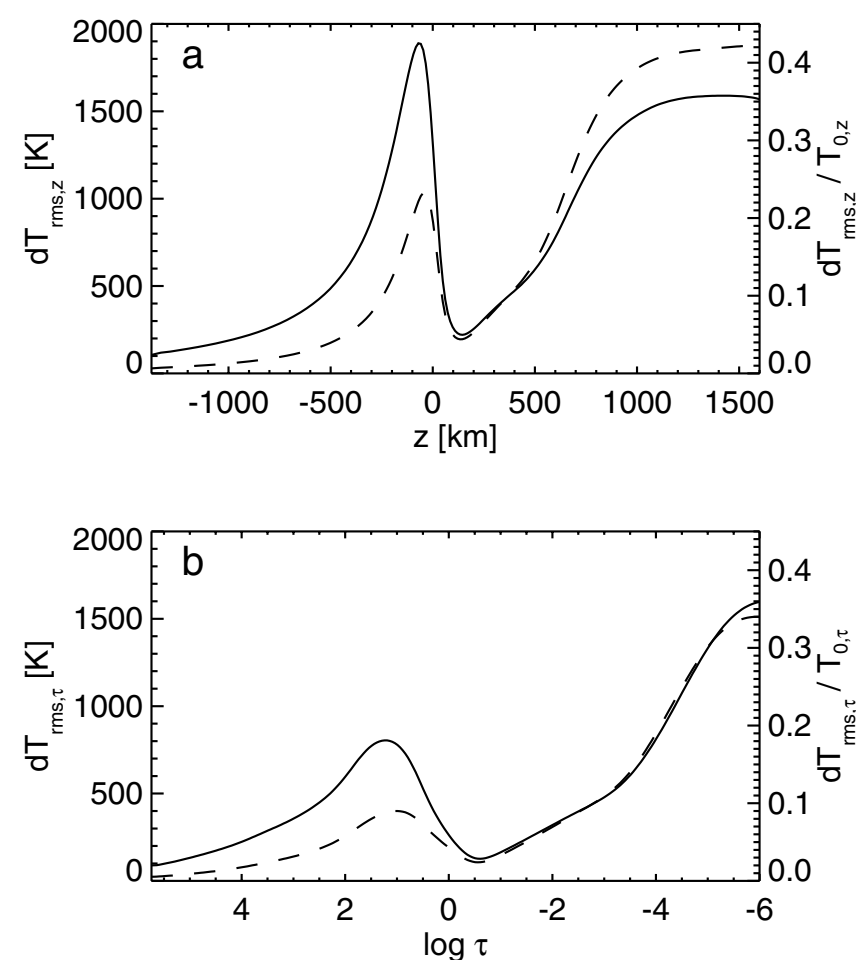

Fig. 9. Horizontally and temporally averaged temperature fluctuation: absolute deviations $\mathrm{d} T_{\mathrm{rms}}$ (solid, left axis) and relative deviations $\mathrm{d} T_{\mathrm{rms}} / T_{0}$ (dashed, right axis) on the geometrical height scale a) and on the optical depth scale b).

Like for the temperature stratification (Sect. 4.4) there is a difference between the geometrical height scale and the optical depth scale. Again the temperature deviations are generally much smaller on a surface of a particular optical depth than for a corresponding geometrical height (see, e.g., Uitenbroek $2000 \mathrm{~b}$ ). In both cases the average lies below $\mathrm{d} T_{\mathrm{rms}} / T_{0} \approx 0.42$. For particular vertical positions and time steps, maximum values of $\approx 1.0$ can be reached.

A comparable quantity $\delta T / T$ has been used by Kalkofen (2001) to distinguish between the two opposing cases of a hot chromosphere with small temperature fluctuations $(\delta T / T \approx 0.1)$ and a cool one with large fluctuations $(\delta T / T \approx 10)$. Our model lies in between these cases. As mentioned earlier, the inclusion of time-dependent ionisation likely leads to higher temperature peaks and accordingly to larger temperature deviations.

\subsection{Cool regions}

As a consequence of the propagating shock waves, the temperature at a fixed position in the model chromosphere varies by several $1000 \mathrm{~K}$ with time, featuring sharp temperature peaks on top of a cool background (Fig. 10c). Observations of the infrared $\mathrm{CO}$ fundamental vibration-rotation lines imply temperatures as low as $3700 \mathrm{~K}$ (e.g., Uitenbroek 2000a) which also represent a lower limit for the average temperature stratification of the 3D model (see Sect. 4.4). We adopt this temperature as a threshold value and determine how long the temperature at a fixed position in the model stays below this value.



Fig. 10. Variation of temperature with time for single grid cells at different heights. In panel c) the cool episodes (time intervals with $T<$ $3700 \mathrm{~K})$ are marked with horizontal bars, together with the duration in seconds.

In the following we will refer to these time intervals with $T<T_{\text {thres }}=3700 \mathrm{~K}$ as cool episodes. In Fig. 10c such episodes are illustrated. The duration of a cool episode is influenced by the local background temperature and the temperature fluctuations due to the propagating waves. Therefore, it depends on height. However, the average duration stays more or less constant throughout a wide height range in the chromosphere. For the 3D model, we determined the average duration of the cool episodes in the chromosphere to be 70-100 s (Fig. 11a). In some cases the cool episodes are much longer, up to several hundred seconds.

With regard to a more global view of the chromosphere not only the duration of single cool episodes but also the sum of all durations is interesting. The temperature at a fixed position in the chromosphere of the 3D model stays roughly half of the time below $T_{\text {thres }}=3700 \mathrm{~K}$ (Fig. 11b). In the lower photosphere cool episodes are rare and thus negligible with regard to the total time.

The spatial scales of the cool regions might also be interesting for the interpretation of observations. The average radius of a cool region is hard to determine because the regions are often not closed structures like a cloud but are connected to other cool regions in a complicated way. As can be seen from Fig. 2 the spatial scales are on average comparable to the granulation, except for some rare cases with larger cool areas. The fraction of the integrated cool area at a particular height shows only relatively small temporal fluctuations (Fig. 12a). Thus, the model chromosphere is never completely cold and never completely hot. There are always cool regions next to a hot component. The height-dependent time-average of the cool area fraction (see Fig. 12b) is equal to the average ratio of cool time to total time 

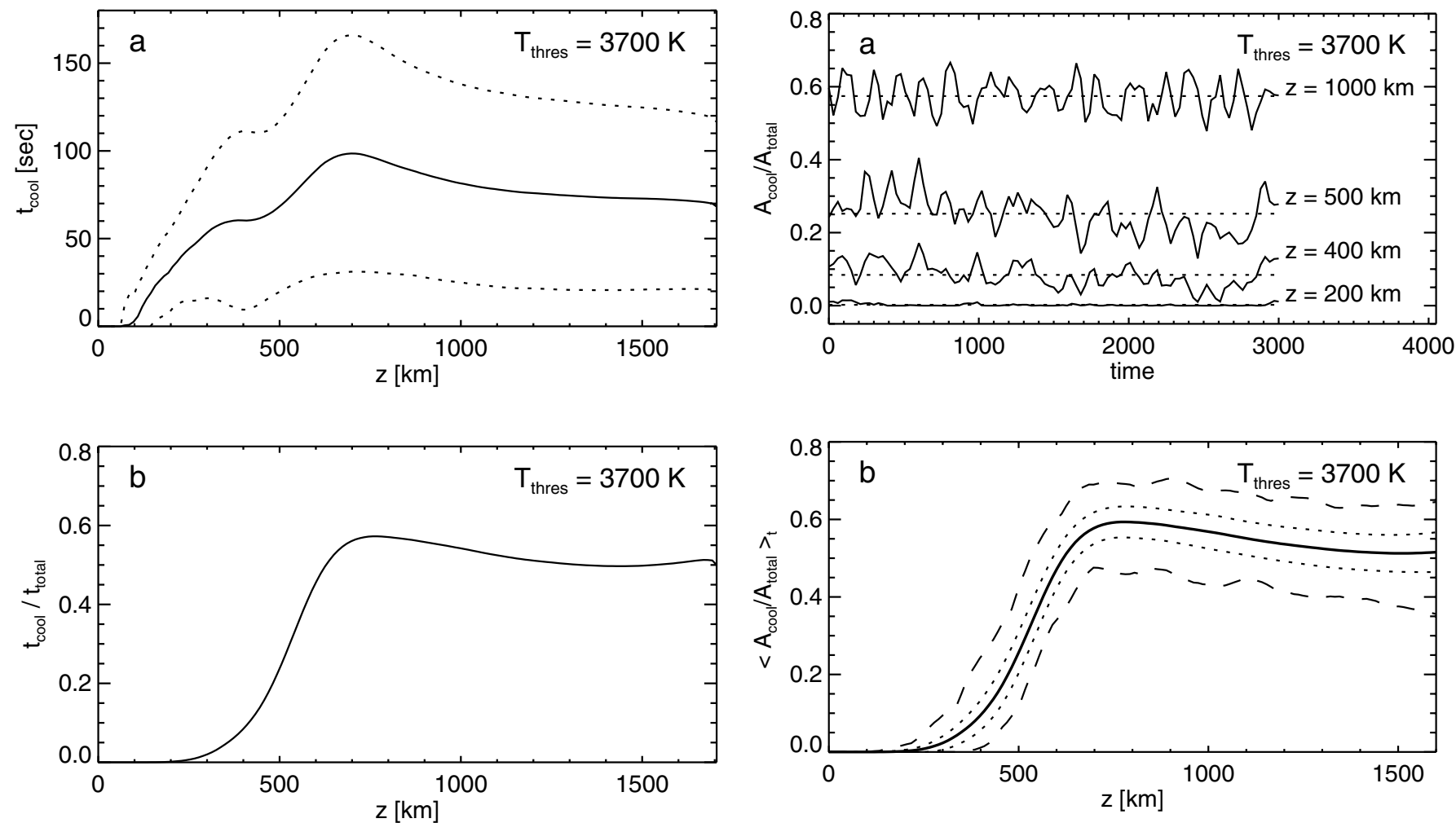

Fig. 11. Height-dependent duration of cool episodes for a threshold temperature of $T_{\text {thres }}=3700 \mathrm{~K}$ in the 3D model. a) Absolute values for the average (solid) and the average plus/minus standard deviation (dotted); b) Ratio of integrated cool time to total time.

(Fig. 11b) because both represent the horizontally and temporally averaged number of grid cells with temperatures below the threshold value. On average 50 to $60 \%$ of the whole time and of the whole area in a horizontal slice of the model chromosphere has a temperature below $3700 \mathrm{~K}$. Consequently, this cool component is not just a minor constituent in our 3D model.

\subsection{Carbon monoxide}

It is not obvious how the variable hydrodynamic conditions affect the formation, dissociation, and spatial distribution of $\mathrm{CO}$ molecules in the outer solar layers. Here we present the results of a simple time-dependent calculation of the $\mathrm{CO}$ concentration, demonstrating that the predicted height distribution of $\mathrm{CO}$ can be very different in a static and in a dynamic solar atmosphere, because the reaction rates are highly non-linear functions of temperature.

For simplicity, we assume that $\mathrm{CO}$ is formed by direct radiative association, $\mathrm{C}+\mathrm{O} \rightarrow \mathrm{CO}+h v$, and is destroyed by collisional dissociation, $\mathrm{CO}+\mathrm{H} \rightarrow \mathrm{C}+\mathrm{O}+\mathrm{H}$. In this case, the temporal evolution of the $\mathrm{CO}$ concentration $[\mathrm{CO}]$ is governed by the differential equation

$$
\frac{\mathrm{d}}{\mathrm{d} t}([\mathrm{CO}])=k_{1}-k_{2}[\mathrm{CO}]
$$

where $[\mathrm{CO}]=n_{\mathrm{CO}} /\left(n_{\mathrm{C}}+n_{\mathrm{CO}}\right)$; a value of 1 means that all carbon is bound in $\mathrm{CO}$ molecules. According to Ayres \& Rabin (1996), the constants $k_{1}$ and $k_{2}$ depend on the

Fig. 12. Ratio of cool area to total area for a threshold temperature of $T_{\text {thres }}=3700 \mathrm{~K}$ in the $3 \mathrm{D}$ model. a) Variation with time (solid) and time-averages (dotted) at different heights; b) Height-dependent time-average (solid), \pm standard deviation (dotted), and maximum and minimum values (dashed).

number density of neutral hydrogen, $n_{\mathrm{H}}$, and on the temperature $T$ as

$k_{1}=2.5 \times 10^{-5} n_{15} \tilde{T}^{0.6}$

and

$k_{2}=k_{1}\left(1+40 \tilde{T}^{22.2}\right)$

with the notations $n_{15}=n_{\mathrm{H}} /\left(10^{15} \mathrm{~cm}^{-3}\right)$ and $\tilde{T}=T /(5000 \mathrm{~K})$.

In a static environment, the equilibrium $\mathrm{CO}$ concentration,

$[\mathrm{CO}]_{\mathrm{eq}}=\frac{k_{1}}{k_{2}}=\left(1+40 \tilde{T}^{22.2}\right)^{-1}$,

is approached with a characteristic time scale

$t_{\mathrm{CO}}^{\text {chem }}=k_{2}^{-1}=\frac{410^{4}}{n_{15}} \frac{\tilde{T}^{-0.6}}{1+40 \tilde{T}^{22.2}}[\mathrm{~s}]$.

The characteristic time scale $t_{\mathrm{CO}}^{\text {chem }}$ according to Eq. (7) and the equilibrium $\mathrm{CO}$ concentration $[\mathrm{CO}]_{\mathrm{eq}}$ according to Eq. (6) are plotted as a function of height for the mean temperature and density structure of our 3D simulation in Fig. 13 (thin solid lines). $t_{\mathrm{CO}}^{\text {chem }}$ varies by orders of magnitude, from $\approx 0.1 \mathrm{~s}$ at $z=0 \mathrm{~km}(\tau \approx 1)$ to $\gtrsim 10^{6} \mathrm{~s}$ at $z=1000 \mathrm{~km}$. This variation is partly due to the temperature dependence of $t_{\mathrm{CO}}^{\mathrm{chem}}$, but mainly due to the density factor. In the lower chromosphere ( $z \gtrsim 600 \mathrm{~km}$ ), [CO] $\gtrsim 0.9$, implying that almost all carbon in the chromosphere is bound in $\mathrm{CO}$. 

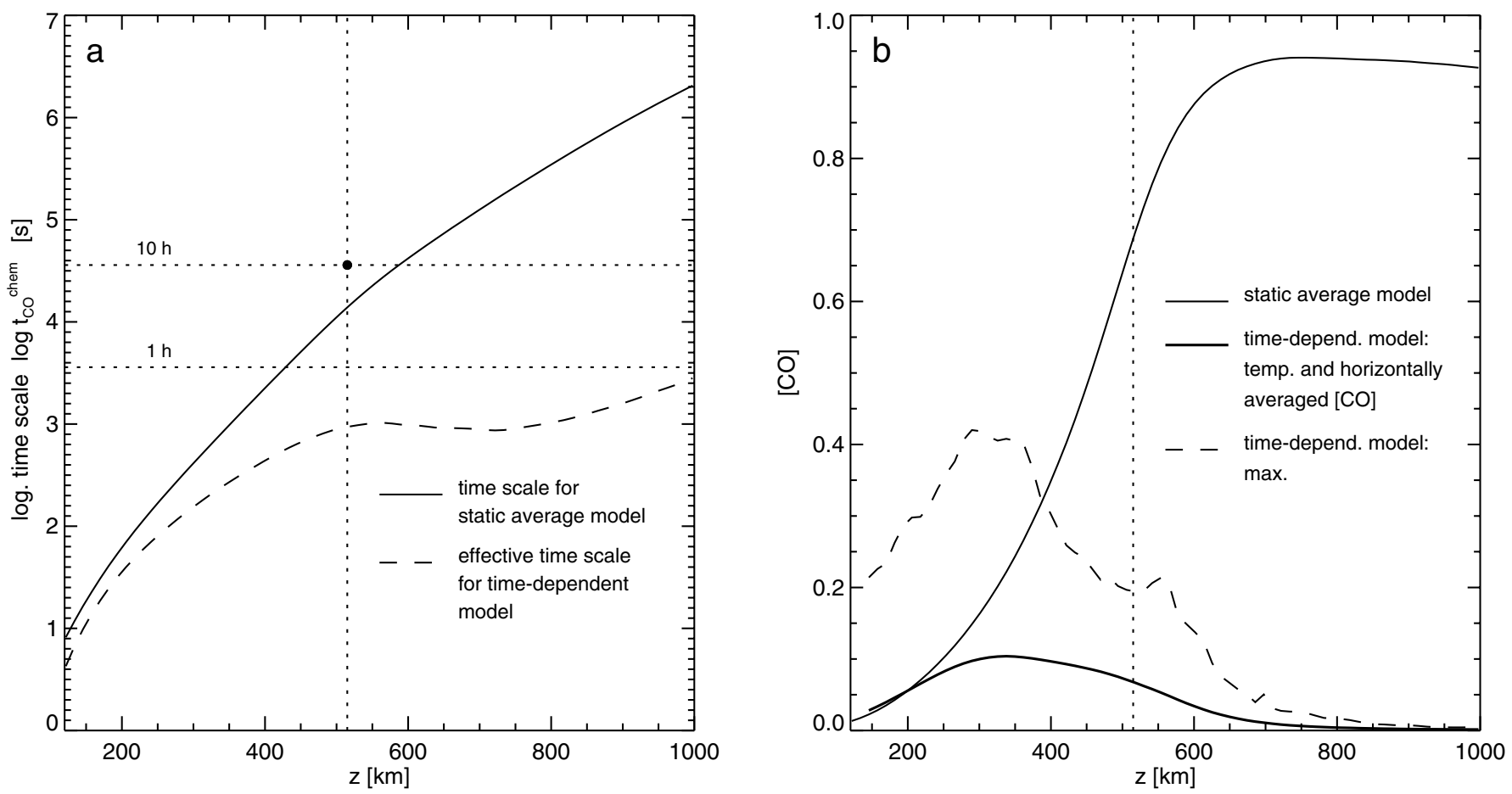

Fig. 13. Formation and destruction of carbon monoxide. a) Chemical time scale $t_{\mathrm{CO}}^{\text {chem }}$ for the averaged 3D stratification (solid) and for the timedependent calculation $t_{\mathrm{CO}}^{\text {chem dyn }}$ (dashed). The black dot indicates a time scale of 10 hours at the classical temperature minimum (Ayres $\&$ Rabin 1996). b) Equilibrium CO concentration for the averaged 3D stratification (thin solid), and average CO concentration of the time-dependent calculation (thick solid) together with the corresponding maximum and minimum values (dashed). [CO] $=1$ means all carbon is bound in CO molecules.

The situation is quite different in a dynamic atmosphere. We have investigated the time-dependent case quantitatively, using 196 representative vertical columns of the 3D model described before. The simulations then provide the temperature and density variations at each point for the time interval of $151 \mathrm{~min}$. These prescribed fluctuations translate into timedependent coefficients $k_{1}$ and $k_{2}$ according to Eqs. (4) and (5). We have solved Eq. (3) with these time-dependent coefficients for each point in the selected columns, using a standard fourthorder Runge-Kutta method. If necessary, the time-sequences from the simulations are repeated until a (dynamic) equilibrium is obtained.

The resulting horizontally and temporally averaged $\mathrm{CO}$ concentration is shown in Fig. 13b (thick solid line). It differs dramatically from the distribution found in the static mean atmosphere (thin solid line), except for the deep photosphere $(z \lesssim 200 \mathrm{~km})$. In the dynamical atmosphere, CO is present in the photosphere and in the low chromosphere with a maximum concentration of only $\langle[\mathrm{CO}]\rangle_{t} \approx 0.10$ at $z \approx 340 \mathrm{~km}$. Very little $\mathrm{CO}$ is found in the layers above $z \approx 700 \mathrm{~km}$.

This finding is in line with the calculations by Asensio Ramos et al. (2003) which are based on the 1D numerical simulations by CS. They, too, state that no significant $\mathrm{CO}$ concentration should be present at heights greater than $\approx 700 \mathrm{~km}$. Furthermore, the difference in the $\mathrm{CO}$ concentration between the static and the dynamic approach becomes larger with increasing height (see Fig. 2 in Asensio Ramos et al. 2003) which is qualitatively similar to our results (see Fig. 13b). Hence, we agree with Asensio Ramos et al. that detailed nonequilibrium $\mathrm{CO}$ chemistry must be taken into account.

The reason for the difference between the static and the dynamic model presented in this work, however, is related to the fact that $\mathrm{CO}$ is rather efficiently destroyed during the passage of high-temperature regions (shock fronts), where the chemical reaction time scales are short. In the subsequent cool phases (see also Sect. 4.6), reaction time scales are much longer, so the $\mathrm{CO}$ concentration builds up rather slowly and reaches only moderate levels before the next high-temperature event occurs. The low CO concentration in the upper atmosphere is thus a consequence of the onset of shock formation and the related higher temperature peaks (see Fig. 7a).

If chemical time scales are short compared to the hydrodynamical time scales, $t_{\mathrm{CO}}^{\text {chem }} \ll t_{\mathrm{HD}}$, then chemical equilibrium is reached instantaneously, and $[\mathrm{CO}]_{\mathrm{eq}}^{\mathrm{dyn}} \approx\left\langle k_{1} / k_{2}\right\rangle_{t}$. Hence, we find that the $\mathrm{CO}$ concentration in the lower photosphere $(z \lesssim 200 \mathrm{~km})$ is reasonably well represented by this approximation. In these layers, the spatial CO distribution is tightly correlated with the local temperature: the coolest regions have the highest $\mathrm{CO}$ concentration.

For the higher layers $(z \gtrsim 200 \mathrm{~km})$, which are of more interest in this investigation, we find $t_{\mathrm{CO}}^{\text {chem }}>t_{\mathrm{HD}}$. The $\mathrm{CO}$ concentration is then well approximated by $[\mathrm{CO}]_{\mathrm{eq}}^{\mathrm{dyn}} \approx\left\langle k_{1}\right\rangle_{t} /\left\langle k_{2}\right\rangle_{t}$. Since $k_{2}$ is a highly non-linear function of $T$, the hightemperature events vastly dominate the time average and the resulting $\mathrm{CO}$ concentration is much smaller than implied by the mean temperature. 
We can also conclude that the dynamical equilibrium $\mathrm{CO}$ concentration is attained on a characteristic time scale $t_{\mathrm{CO}}^{\text {chem,dyn }}=\left\langle k_{2}\right\rangle_{t}^{-1}$, which is also shown in Fig. 13a (dashed). The correlation between [CO] and $T$ is expected to be poor in the higher layers: the highest concentrations build up in places with the longest history of relatively undisturbed conditions $([\mathrm{CO}] \approx 0.4)$, and almost no $\mathrm{CO}$ is found just behind strong shock fronts.

The results described above can only be a first estimate of the height profile of $[\mathrm{CO}]$ under time dependent conditions, because the underlying calculations still have severe limitations. More secure conclusions about the $\mathrm{CO}$ distribution in the upper solar atmosphere have to wait for more detailed future simulations taking into account (i) the transport of $\mathrm{CO}$ molecules with the flow, (ii) a more complete chemical reaction network including multi-step reactions affecting the $\mathrm{CO}$ balance (see Ayres \& Rabin 1996; Asensio Ramos et al. 2003), and (iii) the back reaction of the $\mathrm{CO}$ concentration on the radiative cooling rate.

\section{Discussion}

Quantitatively, the results presented in the preceding sections must be considered as preliminary, since the physics of $\mathrm{CO}^{5} \mathrm{BOLD}$ are not yet properly adapted to chromospheric conditions. In particular, the assumption of LTE is a poor approximation in the chromosphere (Carlsson \& Stein 2002; Rammacher \& Ulmschneider 2003). A realistic treatment should account for deviations from LTE and also requires the time-dependent computation of the ionisation of hydrogen and other important species. Nevertheless, we believe that some of the basic features seen in our model are insensitive to the detailed treatment of thermodynamics and radiative transfer.

The 3D topology of the small-scale chromospheric network we discovered in our simulation, and its spatial and temporal scales are expected to be a robust feature. This is confirmed by test calculations with different values of the tensor viscosity, a different grey opacity table, and even with a frequencydependent (multi-group) radiative transfer scheme using five opacity bins: the dynamical properties of these models (like the height-dependent amplitude of the velocity fluctuations) turn out to be quite insusceptible to changes of the analysed numerical parameters. We attribute this to the fact that the dynamics of the model chromosphere are governed by the lower layers where the excitation of acoustic waves takes place and that the numerical modelling of these layers, i.e., the photosphere and the top of the convection zone, is quite realistic. In this context, it is reassuring to find prominent chromospheric oscillations in the 3-min range whose properties are largely independent of the numerical details of the simulation. The qualitative similarity to observations indicates that the dynamics are indeed modelled reasonably well.

The horizontal structure of our model chromosphere, i.e., its topology, is reminiscent of observed patterns like the chromospheric "background pattern" found by (Krijger et al. 2001) and the structure of Ca II H observations (Sütterlin 2003). The latter, for instance, exhibits spatial scales which are comparable to the granulation and thus to the scales which are found in our numerical simulation. However, the observed patterns originate predominantly from lower layers and should therefore not be confused with the patterning of the model chromosphere. The differences might be revealed by determining the time scales on which the different patterns evolve. This issue needs to be investigated more properly in the future.

In contrast to the spatial scales and the topology of the atmospheric patterns, the amplitude of the temperature fluctuations in the model chromosphere is more susceptible to the treatment of radiative transfer. Indeed, the temperature fluctuations are significantly smaller in the aforementioned test calculation using a frequency-dependent radiative transfer scheme. However, we recall that, up to the mid-chromospheric layers $(z=1000 \mathrm{~km})$, the peak shock temperatures in our grey simulation are very similar to those found by Carlsson \& Stein (1994, 1997) and by Skartlien (1998). More precisely, in the lower and mid chromospheric regions CS find peak temperatures which lie only somewhat ( 1000 K) above our values.

The shock peak temperatures are of importance since many spectral features are biased towards high temperatures. Theoretically, the peak temperatures depend on the shock strength and are given by the Rankine-Hugoniot jump conditions. In the absence of radiation, a conservative numerical scheme guarantees that the jump conditions are fulfilled, i.e., the post-shock temperature is independent of the spatial resolution. If radiation is important, however, the peak post-shock temperature is reduced relative to the theoretical value by an amount that depends on the spatial resolution of the numerical grid. This is because the shock heating is stretched out over a finite time interval, given by the time a volume element needs to cross the shock front which is smeared out over a number of grid points. In this case, radiative cooling can reduce the attainable peak temperature if the radiative cooling time is comparable or smaller than the time scale of shock heating. Furthermore, the overall energy dissipation in the shock is altered due to a change of the effective adiabatic exponent of the gas.

In our model based on grey radiative transfer all chromospheric layers are optically thin. Here, radiative cooling times are independent of the flow geometry and mainly dependent on temperature. The cooling time at a temperature of $7000 \mathrm{~K}-$ about the highest temperature we observe in the simulation amounts to $\sim 200 \mathrm{~s}$, and is increasing rapidly for lower temperatures. The dissipation time scale in the shocks is in the order of a few seconds. This means that the thermal structure of our shocks is hardly affected by radiation and primarily given by the shock strength. Only in the most extreme cases we expect some limiting influence of radiative cooling on the post-shock temperature. Similarly, the thermal structure of the post-shock regions is mainly controlled by cooling via adiabatic expansion.

As mentioned above, the differences in the peak temperatures of our model and the simulation by CS become larger in the higher layers. First, CS employ an adaptive grid in their simulation with a grid spacing of typically $200 \mathrm{~m}$ near shocks which is thus much finer than our fixed (vertical) spacing of $12 \mathrm{~km}$. Furthermore, it appears plausible that our radiative transfer - based on Rosseland opacities including lines as 
true absorption - produces shorter radiative cooling times compared to CS. The higher resolution and longer radiative cooling times in the model of CS lead us to expect that their shock peak temperatures are also largely unaffected by radiation.

Two effects can explain the somewhat higher shock temperatures of CS. First, the shock strength in the CS model might simply be higher than in our case. This could be related to the semi-empirical piston velocity CS feed in at the bottom of their model, or their 1D geometry forcing shocks to remain plane-parallel. As we have seen above, extended horizontal shocks are more the exception than the rule in our 3D simulation; most shock fronts weaken as they propagate radially away from their source. Another effect is related to our assumption that thermodynamic equilibrium conditions prevail in the chromosphere. In a recent paper, Carlsson \& Stein (2002) demonstrated that this is a poor approximation. Ionisation equilibria cannot follow the rapid thermodynamic changes introduced by the flow. One consequence is that the energy which is dissipated in shocks cannot go into ionisation but has to go into a temperature increase of the post-shock gas. Moreover, accounting for finite recombination time scales instead of assuming ionisation equilibrium could reduce the ability of the postshock gas to cool, thus leading to even higher temperatures. Since CS account for the thermodynamic non-equilibrium effects, their shock temperatures should be higher.

We cannot decide on the basis of the available information which is the reason for the differences in the peak temperatures of the shocks. However, we conclude that the differences up to the mid-chromospheric layers $(z=1000 \mathrm{~km})$ are modest: our peak temperatures are $\approx 7000 \mathrm{~K}$ compared to $\approx 8000 \mathrm{~K}$ in the CS model. We further note that the chromospheric peak temperatures found by Skartlien (1998) (see his Fig. 9) are $\$ 7000 \mathrm{~K}$ in their case of frequency-dependent radiative transfer accounting for line scattering, which is surprisingly close to our result obtained with our grey LTE radiative transfer.

This supports our conclusion that our grey radiative transfer employed in this work is more realistic than the frequencydependent method available for $\mathrm{CO}^{5} \mathrm{BOLD}$. The latter method strongly overestimates the (LTE) cooling in the strong spectral lines (treated as true absorption) and thus wrongly reduces the maximum attainable temperatures. However, note that also the grey radiative transfer is not appropriate for chromospheric conditions. Rather, a detailed frequency-dependent non-LTE radiative transfer is necessary. Furthermore, for a quantitative comparison with the observations it would be necessary to perform three-dimensional spectrum synthesis, which is planned for the future.

In contrast to the peak temperatures, the mean chromospheric temperature (and also the minimum temperatures) in our simulation are significantly lower than those found by CS (see Fig. 8), and also somewhat cooler than in the Skartlien (1998) model. Obviously, the mean temperature structure is more strongly influenced by the treatment of radiative transfer than it is the case for the peak temperatures. It should therefore be considered as uncertain. Somewhat surprisingly, however, we note that our grey and our frequency-dependent simulations produce almost identical mean chromospheric temperature structures.

Nevertheless, the differences in the average temperature stratification between the one-dimensional simulation by CS and the presented 3D model can be understood if one watches the velocity field of a region which just has been traversed by a strong shock wave. The flows are mostly directed outwards away from the centre of such a region. We interpret this as fast and thus adiabatic expansion of the traversed region. This "dynamic cooling" is obviously more efficient in 3D than 1D simply due to the additional spatial dimensions. This effect thus produces lower average and minimum temperatures in our simulations compared to those of CS.

Furthermore, we point out that the thermal bifurcation in our 3D model (see Sect. 4.3) is not due to the action of carbon monoxide as a cooling agent. Rather, it is caused by the acoustic wave field and the resulting dynamic cooling of adiabatically expanding regions as discussed above. Carbon monoxide is only taken into account in the grey opacity tables so far, and so its real influence is underestimated. A similar simulation with a different grey opacity table without molecular contributions (based on ATLAS6, Kurucz 1970) leads to very similar results. We thus conclude that $\mathrm{CO}$ plays no active role in our present simulations. On the other hand, the calculations described in Sect. 4.7 demonstrate that there is a non-negligible amount of $\mathrm{CO}$ present in the lower chromosphere. Hence, a full treatment of $\mathrm{CO}$ as a cooling agent might even amplify the thermal bifurcation of the chromosphere (see, e.g., Ayres 1981; Anderson \& Athay 1989; Steffen \& Muchmore 1988).

Although the mean chromospheric temperature of our simulation lies considerably below the radiative equilibrium temperature, we find a net radiative cooling of the chromospheric layers: $\left\langle\nabla \cdot \boldsymbol{F}_{\text {rad }}\right\rangle_{\mathrm{t}}$ is positive here. This apparent contradiction can be explained by the presence of sufficiently strong temperature fluctuations and the highly non-linear temperature dependence of the radiative heating/cooling rates. We do not claim, however, that this situation is actually realized in the solar chromosphere.

Our simulations indicate that the wave generation is mainly controlled by the large-scale dynamical evolution of the granulation pattern (see Sect. 4.2). This is at variance with the classical picture of the Lighthill-Stein theory (Lighthill 1952; Stein 1967, 1968) where small-scale turbulent eddies make the main contribution to the acoustic energy flux. Applying the LighthillStein theory to the Sun, Musielak et al. (1994) find that the acoustic flux spectrum shows a maximum near $v \gtrsim 15 \mathrm{mHz}$, and hence is dominated by "short period waves".

The presented simulation can marginally resolve turbulent eddies in the convection zone with wavenumbers up to $k_{\max } \approx 2 \pi /(5 \Delta x)$. According to the classical theory, eddies with wavenumber $k$ mostly contribute to the acoustic wave spectrum at frequency $\omega=k u_{k}$, where $u_{k}$ is the turbulent velocity of eddies with wavenumber $k$. Since $u_{k} \lesssim 1 \mathrm{~km} \mathrm{~s}^{-1}$, the simulation cannot describe the turbulence spectrum beyond $\omega_{\max } \lesssim 30 \mathrm{mHz}, v_{\max } \lesssim 5 \mathrm{mHz}$. We conclude that our present model cannot resolve the small-scale turbulence which is responsible for the sound generation in the LighthillStein theory. The acoustic flux resulting from our simulation 
decreases monotonically with frequency, and so has little in common with the spectrum predicted by the Lighthill-Stein spectrum. Hence, it appears doubtful whether the classical theory, based on the assumption of isothermal, homogeneous, and isotropic turbulence, captures the essential physics of the violent, highly anisotropic layers at the top of real stellar convection zones. We argue that our numerical simulation correctly represents the basic mode of wave generation, even at the present spatial resolution.

\section{Conclusions}

Based on a detailed 3D simulation of the solar granulation and the overlying atmosphere, we have studied the generation of waves by the time-dependent convective flow, and the wave propagation and dissipation in the higher layers. The most important improvements compared to previous numerical simulations are (i) self-consistent dynamics without a need for a driving piston like done by $\mathrm{CS}$ and (ii) a high spatial resolution which is obviously necessary for modelling the small-scale structure of the solar chromosphere. On the other hand, the LTE treatment of the thermodynamics and the radiative transfer is certainly unrealistic in the chromospheric layers. We have presented evidence that some of the basic features seen in our model are nevertheless representative of the (non-magnetic) internetwork regions of the solar chromosphere.

The main result of the present investigation is the discovery of a complex network of hot filaments pervading the otherwise cool chromospheric layers. Caused by interaction of standing and propagating hydrodynamic waves of large amplitude, the model chromosphere is a highly dynamical, spatially and temporally intermittent phenomenon. Its temperature structure is characterised by a thermal bifurcation: hot and cool regions co-exist side by side. Temperatures in the hot filaments are high enough to produce chromospheric emission lines, and the cool "bubbles" are cold enough to form molecular features. Thus, the chromosphere is hot and cold at the same time. This picture of the 3D structure of the solar chromosphere has the potential to explain the apparently contradictory observational diagnostics which cannot be understood in the framework of one-dimensional theoretical or semi-empirical models.

The presence of strong spatial and temporal temperature fluctuations has a remarkable consequence: the temperature minimum and the outward directed temperature rise inferred from semi-empirical models might be artifacts in the sense that they do not necessarily imply an increase of the average gas temperature with height. Our model suggests that the radiative emission can be sustained by the hot propagating shock waves even though the main fraction of the chromospheric layers is cool and the mean gas temperature profile shows an almost monotonic decrease - a conclusion already reached by Carlsson \& Stein $(1994,1995,1997)$ on the basis of one-dimensional hydrodynamical simulations.

We conclude that improved 3D radiation hydrodynamic simulations of the kind presented in this work are likely to lead the way towards a consistent physical model of the thermal structure and dynamics of the non-magnetic solar chromosphere which eventually can explain the various observational diagnostics.

Acknowledgements. We are grateful to M. Carlsson, R. F. Stein, M. Wunnenberg, and P. Sütterlin for providing data for comparison and to R. J. Rutten for advice and critical comments. The numerical simulations were carried out on the CRAY SV1 of the Rechenzentrum der Universität Kiel. SW was supported by the Deutsche Forschungsgemeinschaft (DFG), project Ho596/39. HGL acknowledges financial support of the Walter Gyllenberg Foundation (Lund), and the Swedish Vetenskapsrådet. BF is supported by a grant from the Swedish Schönbergs Donation.

\section{References}

Anderson, L. S., \& Athay, R. G. 1989, ApJ, 346, 1010

Asensio Ramos, A., Trujillo Bueno, J., Carlsson, M., \& Cernicharo, J. 2003, ApJ, 588, L61

Asplund, M., Ludwig, H.-G., Nordlund, ^̊., \& Stein, R. F. 2000, A\&A, 359,669

Avrett, E. H. 1995, in Infrared Tools for Solar Astrophysics: What's Next? Proc. 15th NSO Sac Peak Workshop, ed. J. Kuhn, \& M. Penn (Singapore: World Scientific), 303

Ayres, T. R. 1981, ApJ, 244, 1064

Ayres, T. R. 2002, ApJ, 575, 1104

Ayres, T. R., \& Linsky, J. L. 1976, ApJ, 205, 874

Ayres, T. R., \& Rabin, D. 1996, ApJ, 460, 1042

Ayres, T. R., \& Testerman, L. 1981, ApJ, 245, 1124

Ayres, T. R., Testerman, L., \& Brault, J. W. 1986, ApJ, 304, 542

Buchholz, B., Ulmschneider, P., \& Cuntz, M. 1998, ApJ, 494, 700

Carlsson, M., Judge, P. G., \& Wilhelm, K. 1997, ApJ, 486, L63

Carlsson, M., \& Stein, R. F. 1994, in Proc. Mini-Workshop on Chromospheric Dynamics, ed. M. Carlsson (Oslo: Inst. Theor. Astrophys.), 47

Carlsson, M., \& Stein, R. F. 1995, ApJ, 440, L29

Carlsson, M., \& Stein, R. F. 1997, ApJ, 481, 500

Carlsson, M., \& Stein, R. F. 2002, ApJ, 572, 626

Cuntz, M., Rammacher, W., \& Ulmschneider, P. 1994, ApJ, 432, 690

Fontenla, J. M., Avrett, E. H., \& Loeser, R. 1993, ApJ, 406, 319

Freytag, B., Steffen, M., \& Dorch, B. 2002, Astron. Nachr., 323, 213

Hauschildt, P. H., Baron, E., \& Allard, F. 1997, ApJ, 483, 390

Iglesias, C. A., Rogers, F. J., \& Wilson, B. G. 1992, ApJ, 397, 717

Kalkofen, W. 2001, ApJ, 557, 376

Krijger, J. M., Rutten, R. J., Lites, B. W., et al. 2001, A\&A, 379, 1052

Kurucz, R. L. 1970, SAO Special Report, 308

Lighthill, M. J. 1952, Proc. R. Soc. Lond., A 211, 564

Ludwig, H.-G., Allard, F., \& Hauschildt, P. H. 2002, A\&A, 395, 99

Maltby, P., Avrett, E. H., Carlsson, M., et al. 1986, ApJ, 306, 284

Muchmore, D., \& Ulmschneider, P. 1985, A\&A, 142, 393

Muglach, K., \& Schmidt, W. 2001, A\&A, 379, 592

Musielak, Z. E., Rosner, R., Stein, R. F., \& Ulmschneider, P. 1994, ApJ, 423, 474

Nordlund, A., \& Stein, R. F. 2001, ApJ, 546, 576

Noyes, R. W., \& Hall, D. N. B. 1972, BAAS, 4, 389

Quirk, J. 1994, Int. J. for Numerical Methods in Fluids, 18, 555

Rammacher, W., \& Ulmschneider, P. 2003, ApJ, 589, 988

Roe, P. L. 1986, Ann. Rev. Fluid Mech., 18, 337

Rutten, R. G. M., Schrijver, C. J., Lemmens, A. F. P., \& Zwaan, C. 1991, A\&A, 252, 203

Sütterlin, P. 2003, priv. comm.

Schrijver, C. J. 1987, A\&A, 172, 111

Skartlien, R. 1998, Ph.D. Thesis, University of Oslo, Norway

Skartlien, R. 2000, ApJ, 536, 465 
Skartlien, R., Stein, R. F., \& Nordlund, Å. 2000, ApJ, 541, 468

Solanki, S. K., Livingston, W., \& Ayres, T. 1994, Science, 263, 64 Steffen, M., \& Muchmore, D. 1988, A\&A, 193, 281

Stein, R. F. 1967, Sol. Phys., 2, 385

Stein, R. F. 1968, ApJ, 154, 297

Stein, R. F., \& Nordlund, A. 1998, ApJ, 499, 914

Stein, R. F., \& Nordlund, ̊̊. 2001, ApJ, 546, 585

Uitenbroek, H. 2000a, ApJ, 531, 571

Uitenbroek, H. 2000b, ApJ, 536, 481

Uitenbroek, H., Noyes, R. W., \& Rabin, D. 1994, ApJ, 432, L67

Ulmschneider, P. 1971, A\&A, 12, 297

Ulmschneider, P. 1989, A\&A, 222, 171

Ulmschneider, P., Fawzy, D., Musielak, Z. E., \& Stępień, K. 2001, ApJ, 559, L167
Ulmschneider, P., \& Kalkofen, W. 1977, A\&A, 57, 199

Ulmschneider, P., Muchmore, D., \& Kalkofen, W. 1987, A\&A, 177, 292

Ulmschneider, P., Schmitz, F., Kalkofen, W., \& Bohn, H. U. 1978, A\&A, 70, 487

Ulmschneider, P., Theurer, J., \& Musielak, Z. E. 1996, A\&A, 315, 212

Ulmschneider, P., Theurer, J., Musielak, Z. E., \& Kurucz, R. 1999, A\&A, 347, 243

Vernazza, J. E., Avrett, E. H., \& Loeser, R. 1981, ApJS, 45, 635

Wedemeyer, S. 2003, Ph.D. Thesis, University of Kiel,

http://e-diss.uni-kiel.de/diss_764/

Wiedemann, G., Ayres, T. R., Jennings, D. E., \& Saar, S. H. 1994, ApJ, 423, 806

Wunnenberg, M., Kneer, F., \& Hirzberger, J. 2002, A\&A, 395, L51 